\title{
LIVER AND HEART MITOCHONDRIA OBTAINED FROM ADELIE PENGUIN (PYGOSCELIS ADELIAE) OFFERS HIGH RESISTANCE TO LIPID PEROXIDATION
}

\author{
Mariana Gavazza, ${ }^{1}$ Mónica Marmunti, ${ }^{1}$ \\ D. Montalti ${ }^{2}$ and AnA María GutiérReZ ${ }^{1,2 *}$ \\ ${ }^{1}$ Cátedra Bioquímica, Facultad de Ciencias. Veterinarias, \\ ${ }^{2}$ Cátedra de Fisiología Animal, Facultad de Ciencias Naturales y Museo, \\ Universidad Nacional de La Plata, La Plata, Argentina
}

(Received: November 2, 2006; accepted: June 7, 2007)

\begin{abstract}
Lipid peroxidation is generally thought to be a major mechanism of cell injury in aerobic organisms subjected to oxidative stress. All cellular membranes are especially vulnerable to oxidation due to their high concentration of polyunsaturated fatty acids. However, birds have special adaptations for preventing membrane damage caused by reactive oxygen species. This study examines fatty acid profiles and susceptibility to lipid peroxidation in liver and heart mitochondria obtained from Adelie penguin (Pygoscelis adeliae). The saturated fatty acids in these organelles represent approximately $40-50 \%$ of total fatty acids whereas the polyunsaturated fatty acid composition was highly distinctive, characterized by almost equal amounts of 18:2 n-6; 20:4 n-6 and 22:6 n-3 in liver mitochondria, and a higher proportion of 18:2 n-6 compared to $20: 4$ n- 6 and 22:6n-3 in heart mitochondria. The concentration of total unsaturated fatty acids of liver and heart mitochondria was approximately $50 \%$ and $60 \%$, respectively, with a prevalence of oleic acid C18:1 $\mathrm{n} 9$. The rate $\mathrm{C} 20: 4 \mathrm{n} 6 / \mathrm{C} 18: 2 \mathrm{n} 6$ and the unsaturation index was similar in liver and heart mitochondria; $104.33 \pm 6.73$ and $100.09 \pm 3.07$, respectively. Light emission originating from these organelles showed no statistically significant differences and the polyunsaturated fatty acid profiles did not change during the lipid peroxidation process.
\end{abstract}

Keywords: Penguin - lipid peroxidation - liver - heart-mitochondria

\section{INTRODUCTION}

The polyunsaturated fatty acids (PUFAs) present in biological membranes exhibit the highest sensitivity to oxidative damage. It is accepted that their sensitivity increases as a power function of the number of double bonds per fatty acid molecule [2]. As both oxygen consumption and oxygen free radical production occurs in mitochond-

\footnotetext{
*Corresponding author; e-mail: anamaria_gutierrez@yahoo.com
} 
rial membranes, a low degree of fatty acid unsaturation in these membranes would be advantageous, in terms of oxidative stress because it would decrease the sensitivity to lipid peroxidation [16]. Non-enzymatic lipid peroxidation and formation of lipid peroxides can be initiated by adding ascorbate in the presence of oxygen and either $\mathrm{Fe}^{+3}$ or $\mathrm{Fe}^{+2}$ ions to various tissue preparations such as homogenates, liver mitochondria, hepatic microsomal suspensions and nuclei [1].

Lipid peroxidation proceeds by a chain reaction that includes initiation, propagation and termination. Initiation occurs when an oxidant gives rise to an initiating lipid peroxyl radical (LOO') by reaction with either a lipid (LH) or pre-existing lipid hydroperoxide $(\mathrm{LOOH})$. Propagation is cycled through rounds of $\mathrm{LOO}^{\circ}$ abstraction of the bis-methylene hydrogen atoms of a polyunsaturated fatty acyl chain to generate additional LOO (after $\mathrm{O}_{2}$ addition) which results in the net conversion of lipids to LOOHs. Lipid peroxidation termination involves the reaction of LOO to form non-radical products or the reaction of one $\mathrm{LOO}^{-}$with another terminating radical to generate non-propagating radical species; the first reaction is particularly interesting as it is accompanied by emission of chemiluminescence [19]. The requirement of iron in lipid peroxidation has been demonstrated by Minotti and Aust [12].

The damage to mitochondrial membranes increased, since they are rich in polyunsaturated fatty acids, mainly arachidonic (C20:4 n6) and docosahexaenoic (22:6 n3) acids, that are highly susceptible to lipid peroxidation [8]. Thus measurement of lipid peroxidation is one of the most commonly used assays for radical induced damage [13]. The consequence of peroxidation of unsaturated fatty acids is severe: damage of membrane function, enzymatic inactivation, toxic effects on cellular division, etc. [14]. In the cells, there are efficient defense systems that control lipid peroxidation and make sure the maintenance of cellular integrity and, therefore, an adequate metabolic and functional activity.

Previous studies have shown that the degree of unsaturation of fatty acids and the sensitivity to lipid peroxidation of liver and heart mitochondria from birds are lower when compared with mammals $[7,15]$. Therefore it has been shown that the heart lipids of canaries and parakeets have lower fatty acid double-bond content than those of mice [15]. The Adelie penguin (Pygoscelis adeliae) inhabits the Antarctic region and breeds during the summer in large colonies on remotes islands. The adults feed exclusively at sea and their diet during the breeding season consist almost entirely of krill (Euphausia superba and E. crystalorophias) [3].

The present study aims to compare fatty acid profiles and nonenzymatic lipid peroxidation of mitochondria obtained from liver and heart of Adelie penguin. Therefore, the potential relationships between the fatty acid composition of the food and any changes in those of the tissues were examined. Liver was selected because of its ability of high production of free radicals and heart due to its high oxygen partial pressure. 


\title{
MATERIALS AND METHODS
}

\begin{abstract}
Animals
The samples were obtained from three adult Adelie penguins from Hope Bay, Antarctica Peninsula. The organs were removed and the stomach contents were collected and transferred to our laboratory maintained at $-20^{\circ} \mathrm{C}$. Liver and heart were analyzed, and the rest of the organs were distributed in different laboratories for other studies. The mean body weight of the birds was 4,500 $\mathrm{g}$.
\end{abstract}

\section{Preparation of liver and heart mitochondria}

Mitochondria were prepared as described by Schneider and Hogeboom [17]. The organs were cut into small pieces and washed extensively with $0.15 \mathrm{M} \mathrm{NaCl}$. A homogenate of the tissue was prepared in solution containing $0.25 \mathrm{M}$ sucrose, 10 $\mathrm{mM}$ Tris-HCl pH 7.4, phenyl-methyl-sulfonyl fluoride (PMSF) $0.1 \mathrm{mM}, 3 \mathrm{ml}$ of solution per $g$ of tissue, using the Potter-Elvejhem homogeneizer. The homogenate was spun at $1,000 \times g$, pellet was discarded and the supernatant was spun at $20,000 \times g$ for 10 min to obtain mitochondria. All operations were performed at $4{ }^{\circ} \mathrm{C}$. Mitochondria was stored at $-83{ }^{\circ} \mathrm{C}$ and used within a week of its preparation, after one cycle of freezing and thawing.

\section{Non-enzymatic lipid peroxidation of mitochondria}

Chemiluminescence and lipid peroxidation were initiated by adding ascorbate-Fe $\mathrm{Fe}^{++}$ to mitochondrial preparations [20]. Mitochondria (1 $\mathrm{mg}$ of protein) were incubated at $37{ }^{\circ} \mathrm{C}$ with $0.01 \mathrm{M}$ phosphate buffer $(\mathrm{pH} 7.4), 0.4 \mathrm{mM}$ ascorbate, final volume $1 \mathrm{ml}$. Phosphate buffer is contaminated with sufficient iron to provide the necessary ferrous or ferric iron for lipid peroxidation (final concentration in the incubation mixture was $2.15 \mu \mathrm{M}$ ) [18]. Mitochondrial preparations which lacked ascorbate-Fe ${ }^{++}$ (control) were prepared simultaneously. Chemiluminescence was measured as counts per min in a liquid scintillation analyzer Packard 1900 TR. Membrane light emission was determined over $120 \mathrm{~min}$ period, and recorded as cpm every $10 \mathrm{~min}$ and the sum total of chemiluminescence was used to calculate $\mathrm{cpm} / \mathrm{mg}$ protein.

\section{Measurement of fatty acid composition}

Mitochondrial lipids from samples peroxidized in the presence or in the absence of ascorbic acid and aliquots of stomach content were extracted with chloroform/methanol $(2: 1 \mathrm{v} / \mathrm{v})$ containing $0.01 \%$ butylated hydroxytoluene (BHT) as antioxidant [5]. Fatty acids were transmethylated with $10 \% \mathrm{~F}_{3} \mathrm{~B}$ in methanol at $60{ }^{\circ} \mathrm{C}$ 
Table 1

Fatty acid composition (area \%) of total lipids from liver and heart mitochondria of Adelie penguin

\begin{tabular}{|c|c|c|c|c|c|c|}
\hline \multirow{2}{*}{ Fatty acid } & \multicolumn{3}{|c|}{ Liver mitochondria } & \multicolumn{3}{|c|}{ Heart mitochondria } \\
\hline & Native & Control & Ascorbate- $-\mathrm{Fe}^{++}$ & Native & Control & Ascorbate- $\mathrm{Fe}^{++}$ \\
\hline C18:0 & $17.52 \pm 1.56$ & $18.41 \pm 1.05$ & $18.55 \pm 1.80$ & $19.83 \pm 2.03$ & $22.89 \pm 3.67$ & $20.33 \pm 3.41$ \\
\hline C18:1 n9 & $38.39 \pm 7.96$ & $36.61 \pm 6.17$ & $36.78 \pm 6.32$ & $37.84 \pm 4.04$ & $35.69 \pm 1.65$ & $38.40 \pm 8.18$ \\
\hline $\mathrm{C} 18: 2 \mathrm{n} 6$ & $5.46 \pm 0.80$ & $5.46 \pm 0.98$ & $5.44 \pm 0.81$ & $15.67 \pm 3.84$ & $16.27 \pm 1.47$ & $14.33 \pm 1.50$ \\
\hline $\mathrm{C} 18: 3 \mathrm{n} 3$ & - & - & - & $1.54 \pm 0.13$ & $2.48 \pm 1.11$ & $1.48 \pm 0.55$ \\
\hline $\mathrm{C} 20: 4 \mathrm{n} 6$ & $4.72 \pm 1.72$ & $5.12 \pm 1.83$ & $4.70 \pm 1.85$ & $4.56 \pm 0.87$ & $3.77 \pm 0.65$ & $3.91 \pm 0.68$ \\
\hline $\mathrm{C} 22: 6 \mathrm{n} 3$ & $6.02 \pm 1.26$ & $6.06 \pm 2.24$ & $6.77 \pm 2.28$ & $1.34 \pm 0.78$ & $1.02 \pm 0.34$ & $1.04 \pm 0.62$ \\
\hline Saturated & $48.43 \pm 5.86$ & $47.68 \pm 5.23$ & $49.39 \pm 5.12$ & $38.04 \pm 1.32$ & $45.49 \pm 6.86$ & $40.85 \pm 8.87$ \\
\hline Monounsaturated & $38.39 \pm 7.96$ & $36.61 \pm 6.17$ & $36.78 \pm 6.32$ & $37.84 \pm 4.04$ & $35.69 \pm 1.65$ & $38.40 \pm 8.18$ \\
\hline Polyunsaturated & $16.20 \pm 1.51$ & $16.63 \pm 2.44$ & $16.91 \pm 1.76$ & $23.11 \pm 3.98$ & $23.54 \pm 0.51$ & $20.75 \pm 0.79$ \\
\hline Total unsaturated & $54.59 \pm 6.57$ & $53.24 \pm 4.82$ & $53.69 \pm 5.73$ & $60.95 \pm 0.93$ & $59.24 \pm 1.57$ & $59.16 \pm 8.87$ \\
\hline Saturated/unsaturated & $0.90 \pm 0.20$ & $0.91 \pm 0.19$ & $0.93 \pm 0.16$ & $0.62 \pm 0.03$ & $0.77 \pm 0.10$ & $0.71 \pm 0.24$ \\
\hline
\end{tabular}

Data are given as the mean \pm S.D. of three independent experiments.

$\mathrm{UI}=$ sum of the percentages of each fatty acid multiplied by the number of double bonds. 
for $3 \mathrm{~h}$. Fatty acid methyl esters were analyzed with a GC-14A gas chromatograph (Shimadzu, Kyoto, Japan) equipped with a packed column (1.80 $\mathrm{m} \times 4 \mathrm{~mm}$ i.d.) GP $10 \%$ DEGS-PS on 80/100 Supelcoport. Nitrogen was used as a carrier gas. The injector and detector temperatures were maintained at $250{ }^{\circ} \mathrm{C}$, the column temperature was held at $200{ }^{\circ} \mathrm{C}$. Fatty acid methyl ester peaks were identified by comparison of the retention times with those of standards. All compositions were expressed as \% by area of total fatty acids.

\section{Unsaturation index (UI)}

UI was calculated according to the formula, UI $=$ sum (fatty acid percent) $\times($ number of double bonds) [9].

\section{Protein determination}

Proteins were determined by the method of Lowry et al. [10] using BSA as standard.

\section{Statistical analysis}

Data were expressed as means \pm S.D. Statistical analysis utilized was Student's $t$-test. Statistical criterion for significance was selected at different $p$ values and indicated in each case.

\section{RESULTS}

\section{Fatty acid composition of liver and heart mitochondria}

The saturated long-chain fatty acids present in native liver and heart mitochondria obtained from Adelie penguin were mainly C16:0 and C18:0 in a percentage of approximately $40-50 \%$. The concentration of total unsaturated fatty acids of liver and heart mitochondria was approximately $50 \%$ and $60 \%$, respectively, with a prevalence of oleic acid C18:1 n9. In liver mitochondria the content of polyunsaturated fatty acids decreased in the order $\mathrm{C} 22: 6 \mathrm{n} 3>\mathrm{C} 20: 4 \mathrm{n} 6>\mathrm{C} 18: 2 \mathrm{n} 6$. Whereas in heart mitochondria were in the order C18:2 n6>C20:4 n6>C22:6 n3. The rate C20:4 $\mathrm{n} 6 / \mathrm{C} 18: 2 \mathrm{n} 6$ as well as the unsaturation index in native liver and heart mitochondria was similar (Table 1). 


\section{Comparison of unsaturation index of liver and heart mitochondria with stomach content}

The fatty acid composition of penguin stomach content is shown in Table 2 . The fatty acid profile is characterized by a high percentage of polyunsaturated fatty acids. The unsaturation index (UI) which is an indication of the number of double bonds present in lipids was similar in liver and heart mitochondria; $104.33 \pm 6.73$ and 100.09 \pm 3.07 , respectively, whereas was 2.4 times higher in stomach content, $243.13 \pm 7.62$. The main reason for this difference was the higher $\mathrm{C} 20: 4 \mathrm{n} 6$ and $\mathrm{C} 22: 6 \mathrm{n} 3$ percentages found in the stomach content compared to mitochondria (Tables 1,2).

Table 2

\begin{tabular}{cc}
$\begin{array}{c}\text { Fatty acid composition (area \%) of total lipids } \\
\text { from stomach content of Adelie penguin }\end{array}$ \\
\multicolumn{3}{c}{ Fatty acid } & Area $\%$ \\
\hline C16:0 & $23.83 \pm 1.14$ \\
C16:1 n7 & $7.93 \pm 0.12$ \\
C18:0 & $0.90 \pm 0.10$ \\
C18:1 n9 & $20.53 \pm 1.37$ \\
C18:2 n6 & $1.93 \pm 0.05$ \\
C18:3 n3 & $0.63 \pm 0.06$ \\
C20:4 n6 & $30.37 \pm 0.47$ \\
C22:6 n3 & $14.58 \pm 1.49$ \\
\hline & \\
& \\
Saturated & $24.73 \pm 1.21$ \\
Monounsaturated & $28.47 \pm 1.42$ \\
Polyunsaturated & $47.50 \pm 1.55$ \\
Total unsaturated & $75.96 \pm 0.21$ \\
Saturated/unsaturated & $0.33 \pm 0.02$ \\
UI & $243.13 \pm 7.62$ \\
\hline
\end{tabular}

Data are given as the mean \pm S.D. of three independent experiments.

$\mathrm{UI}=$ sum of the percentages of each fatty acid multiplied by the number of double bonds.

\section{Fatty acid profiles and chemiluminescence of liver and heart mitochondria submitted to lipid peroxidation}

Fatty acid profiles of liver and heart mitochondria incubated $120 \mathrm{~min}$ at $37^{\circ} \mathrm{C}$ were similar when control (without ascorbate) was compared with peroxidized (with ascorbate- $\mathrm{Fe}^{++}$). The main peroxidable fatty acids C18:2 n6, C20:4 $\mathrm{n} 6$ and $\mathrm{C} 22: 6 \mathrm{n} 3$ do no show significant decreases as expected when the lipid peroxidation process occurs. Coincident with this results, light emission-chemiluminescence originating 
from liver and hearth mitochondria was not statistically significant when control and peroxidized samples were compared. Figures 1 and 2 show the chemiluminescence as a function of time measured in liver and heart mitochondria with and without 0.4 $\mathrm{mM}$ ascorbate. Total chemiluminescence produced by liver and heart mitochondria obtained from Adelie penguin (Pygoscelis adeliae) is indicated in Figure 3.

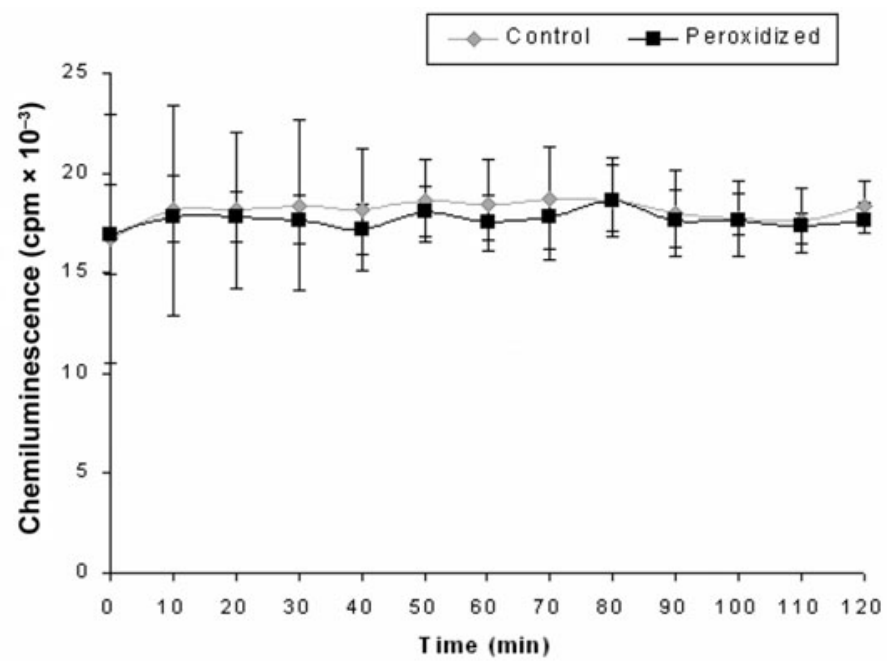

Fig. 1. Chemiluminescence as a function of time during lipid peroxidation of liver mitochondria. Results are expressed as mean \pm S.D. of three independent experiments

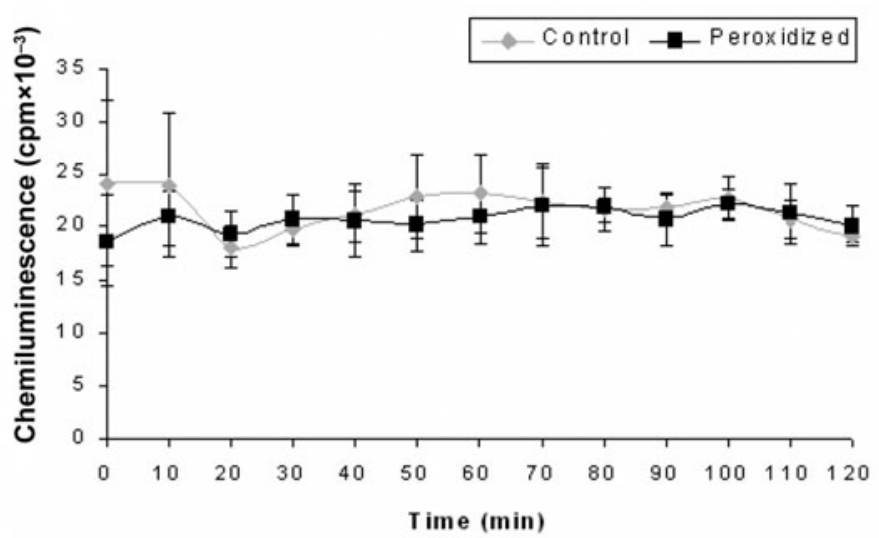

Fig. 2. Chemiluminescence as a function of time during lipid peroxidation of heart mitochondria. Results are expressed as mean \pm S.D. of three independent experiments 


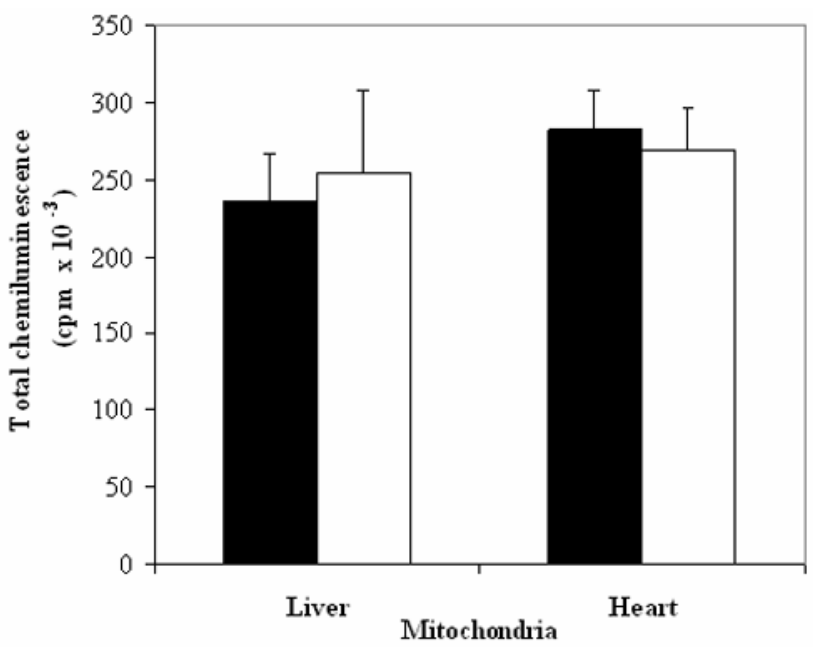

Fig. 3. Total chemiluminescence produced by liver and heart mitochondria obtained from Adelie penguin (Pygoscelis adeliae). $\mathbf{\text { control} ;} \square$ peroxidized (in the presence of ascorbate- $\mathrm{Fe}^{++}$). Results are expressed as mean \pm S.D. of three independent experiments

The fatty acid profiles and unsaturation index of liver and heart mitochondria were not modified after the lipid peroxidation process (Table 1; Figs 1, 2, 3).

\section{DISCUSSION}

In this study we describe for the first time the fatty acid profiles and non-enzymatic lipid peroxidation of mitochondria obtained from liver and heart of Adelie penguin. We demonstrated that although the total unsaturated fatty acids of liver and heart mitochondria were similar in all tissues examined, the contribution of each fatty acid was different. Liver mitochondria posses a lower content of PUFA n6 and a higher content of PUFA $\mathrm{n} 3$ compared with heart mitochondria. These results indicate that the level of unsaturated fatty acids is homeostatically regulated in both tissues [11]. The control of membrane fatty acid unsaturation has been attributed to negative feedback regulation of transcription of desaturase genes dependent on lipid composition [11] and to the modulation of desaturases by the metabolic-hormonal status [6].

Many studies $[7,8,15]$ have demonstrated that different birds species show a low degree of unsaturation despite differences in their diet composition. Taking this observation into account we considered that the low content of double bonds observed in mitochondrial fatty acids in penguins would be independent of diet.

Previous comparative studies of fatty acid composition showed a strong progressive decrease in the total number of double bonds of heart, skeletal muscle and kidney phospholipids as body size increased in five mammals following the order: mouse-rat-rabbit-man-whale [4]. 
To examine this question further, we measured fatty acid composition of liver and heart mitochondria isolated from Adelie penguin. We observed a low content of polyunsaturated fatty acids, the monounsaturated values were similar to those previously reported from birds with high body weight [7]. These findings help to reaffirm the idea that birds possess fatty acids with low degree of unsaturation.

The king penguin is another species that inhabits the sub-Antarctic region and breeds during the summer in large colonies on remote islands. The adults feed exclusively at sea and their diet during the breeding season consists almost entirely of myctophid fish $[21,22]$. This diet is a very rich source of long chain $n-3$ polyunsaturated fatty acids such as 22:6 n-3 and eicosapentaenoic acid (20:5 n-3), and is also characterized by high proportions of long chain monounsaturates including 20:1 n-9, 22:1 n-9, 22:1 n-11 and 24:1 n-9, whereas n-6 polyunsaturates are very minor constituents $[22,23]$. Monounsaturated and saturated fatty acids are much less reactive and do not usually participate in lipid peroxidation. Thus, fatty acids with no double bonds or with 1 double bond can undergo oxidation but not a chain lipid peroxidation process; for example, oleic acid with 18 carbon atoms and 1 double bond (C18:1 n9) cannot undergo the lipid peroxidation process. The amount of fatty acids containing two or more double bonds in liver and heart mitochondria isolated from Adelie penguin is very low to be affected by lipid peroxidation. Mitochondria from both tissues were not sensitive to lipid peroxidation, showing the same pattern of unsaturated fatty acids compared with control. In addition light emission in those organelles did not show significant changes. Potential relationships between the fatty acid composition of the food and any changes in the tissues examined will help to understand how the process of lipid peroxidation affects the mitochondrial membranes.

\section{ACKNOWLEDGEMENT}

We thank Med. Vet. César Arcemis for the excellent technical assistance in performing fatty acid analysis.

\section{REFERENCES}

1. Catalá, A., Cerruti, A. (1997) Non enzymatic peroxidation of lipids isolated from rat liver microsomes, mitochondria and nuclei. Int. J. Biochem. Cell. Biol. 29, 541-546.

2. Catalá, A. (2006) An overview of lipid peroxidation with emphasis in outer segments of photoreceptors and the chemiluminescence assay. Int. J. Biochem. Cell. Biol. 38, 1482-1495.

3. Coria, N. R., Spairani, H., Vivequin, S. M., Fontana, R. (1995) Diet of Adelie penguins Pygoscelis adeliae during the post-hatching period at Esperanza Bay, Antarctica, 1987/88. Polar Biology 15, 415-418.

4. Couture, P., Hulbert, A. J. (1995) Membrane fatty acid composition of tissues is related to body mass of mammals. J. Membr. Biol. 148, 27-39.

5. Folch, J., Lees, N., Sloane Stanley, G. A. (1957) A simple method for the isolation and purification of total lipids from animal tissues. J. Biol. Chem. 226, 497-509. 
6. Guéraud, F., Paris, A. (1997) Hepatic microsomal membrane lipidic composition and growth hormone effect in adult male rat: evidence for a "feminization" process of total phospholipid fatty acid pattern. Biochim. Biophys. Acta 1329, 97-110.

7. Gutiérrez, A. M., Reboredo, C. J., Mosca, S. M., Catalá, A. (2004) Fatty acid composition and lipid peroxidation induced by ascorbate- $\mathrm{Fe}^{2+}$ in different organs of goose (Anser anser). Comp. Biochem. and Physiol C: Toxicol Pharmacol 137, 123-132.

8. Gutiérrez, A. M., Reboredo, G. R., Mosca, S. M., Catalá, A. (2006) A low degree of fatty acid unsaturation leads to high resistance to lipid peroxidation in mitochondria and microsomes of different organs of quail (Coturnix coturnix japonica). Mol. Cell. Biochem. 282, 109-115.

9. Llanillo, M., Sanchez Yague, J., Checa, A., Martín-Valmaseda, E. M., Felipe, A. (1995) Phospholipid and fatty acid composition in stored sheep erythrocytes of different densities. Exp. Hematol. 23, $258-264$.

10. Lowry, O. H., Rosebrough, N. J., Farr, A. L., Randall, R. J. (1951) Protein measurement with the Folin phenol reagent. J. Biol. Chem. 193, 265-275.

11. Maresca, B., Cossins, A. R. (1993) Fatty acid feedback and fluidity. Nature 365, 606-607.

12. Minotti, G., Aust, S. D. (1992) Redox cycling of iron and lipid peroxidation. Lipids 27, 219-226.

13. Ozgova, S., Hermanek, J., Gut, I. (2003) Different antioxidant effects of polyphenols on lipid peroxidation and hydroxyl radicals in the NADPH, Fe-ascorbate and Fe microsomal systems. Biochem. Pharmacol. 66, 1127-1137.

14. Palmer, S. (1994) Antioxidant vitamins and cancer risk. Nutrition 10, 433-434.

15. Pamplona, R., Portero-Otin, M., Ledo, D. F., Gredilla, R., Barja, G. (1999) Heart fatty acid unsaturation and lipid peroxidation, and aging rate, are lower in the canary and the parakeet than in the mouse. Aging Clin. Exp. Res. 11, 44-49.

16. Pamplona, R., Portero-Otin, M., Riba, D., Ruiz, C., Prat, J., Bellmunt, M. J., Barja, G. (1998) Mitochondrial membrane peroxidizability index is inversely related to maximum life span in mammals. J. Lipid. Res. 39, 1989-1994.

17. Schneider, W. C., Hogeboom, H. G. (1950) Intracellular distribution of enzymes. Further studies and distribution of cytochrome $\mathrm{c}$ in rat liver homogenates. J. Biol. Chem. 178, 123-128.

18. Terrasa, A. M., Guajardo, M., Catala, A. (2000) Selective inhibition of the non-enzymatic lipid peroxidation of phosphatidylserine in rod outer segments by $\alpha$-tocopherol. Moll. Cell. Biochem. 211, $39-45$.

19. Vladimirov, Yu., A., Olenev, V. I., Suslova, T. B., Cheremisina, Z. P. (1980) Lipid peroxidation in mitochondrial membrane. Adv. Lipid. Res. 17, 173-249.

20. Wright, J. R., Rumbaugh, R. C., Colby, H. D., Miles, P. R. (1979) The relationship between chemiluminescence and lipid peroxidation in rat hepatic microsomes. Arch. Biochem. Biophys. 192, 344-351.

21. Cherel, Y., Verdon, C., Ridoux, V. (1993) Seasonal importance of oceanic myctophids in King penguin diet at Crozet Islands. Polar Biol. 13, 355-357.

22. Raclot, T., Groscolas, R., Cherel, Y. (1998) Fatty acid evidence for the importance of myctophid fishes in the diet of King penguins, Aptenodytes patagonicus. Mar. Biol. (Berl.). 132, 523-533.

23. Decrock, F., Groscolas, R., McCartney, R. J., Speakew, B. K. (2001) Transfer of n-3 and n-6 polyunsaturated fatty acids from yolk to embryo during development of the King penguin. Am. J. Physiol. Regul. Integr. Comp. Physiol. 280, R843-R853. 